Frauen - Gesellschaft - Kritik

Band 35 


\title{
Die Gärten der Frauen
}

\section{Zur sozialen Bedeutung von Kleinstlandwirtschaft in Stadt und Land weltweit}

\author{
herausgegeben \\ für die Arbeitsgruppe Kleinstlandwirtschaft \\ von Elisabeth Meyer-Renschhausen \\ unter Mitarbeit von Renate Müller und Petra Becker
}

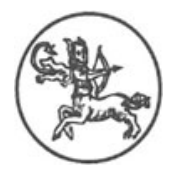

Centaurus Verlag \& Media UG 2002 
Die Deutsche Bibliothek - CIP-Einheitsaufnahme

Bibliographische Information der Deutschen Bibliothek:

Die deutsche Bibliothek verzeichnet diese Publikation in der Deutschen Nationalbibliographie; detaillierte bibliographische Daten sind im Internet über http://dnb.ddb.de abrufbar

ISBN 978-3-8255-0338-3 ISBN 978-3-86226-354-7 (eBook)

DOI 10.1007/978-3-86226-354-7

\section{ISSN 0939-4540}

Alle Rechte, insbesondere das Recht der Vervielfältigung und Verbreitung sowie der Übersetzung, vorbehalten. Kein Teil des Werkes darf in irgendeiner Form (durch Fotokopie, Mikrofilm oder ein anderes Verfahren) ohne schriftliche Genehmigung des Verlages reproduziert oder unter Verwendung elektronischer Systeme verarbeitet, vervielfältigt oder verbreitet werden

(C) CENTAURUS Verlags-GmbH. \& Co. KG, Herbolzheim 2002

Umschlagabbildungen:

Umschlagcover: Protest in New York: „Save the gardens!". Photo: Edie Stone I

Altbäuerin in Osttirol. Photo: Brigitte Vogl-Lukasser /

Frau mit geernteten Zwiebeln (Sudan). Photo: Sigi Grunow,

Süddeutscher Verlag - Bilderdienst.

Umschlagrückseite: Im Schulgarten von Schwedt (Sigrid Fronius und Elisabeth Meyer-Renschausen). Photo: Christophe Kotanyi /

Maria, ihre Schwestern und Verwandte verspeisen die Früchte aus und in

Sigrids Garten. Photo: Sigrid Fronius

Umschlaggestaltung: DTP-Studio, Antje Walter, Hinterzarten

Satz: Centaurus-Satz 


\section{Inhaltsverzeichnis}

Vorwort

IX

\section{WEIBLICHE ÖKONOMIE}

Elisabeth Meyer-Renschhausen

Kleinlandwirtschaft und Gärten als „weibliche Ökonomie“. Eine Einführung.......... 1

Heide Inhetveen

Hortikultur - Abbild der Informellen Ökonomie und Vorbild

für Vorsorgendes Wirtschaften.

Ramesh Chandra Agrawal

Zukunftsperspektiven von Kleinbauern in Entwicklungsländern

Petra Kreinecker

Städtische Landwirtschaft in Bolivien: Eine weibliche Subsistenzstrategie 46

Veronika Bennholdt-Thomsen

Die dörflich-bäuerliche Kultur in der Warburger Börde: Verschüttete Quelle einer eigenständigen regionalen Ökonomie?

\section{ÜBERLEBEN}

Friedhelm Streiffeler

Landwirtschaft als Überlebensbedingung in afrikanischen Städten

Lutgarde Creemers

Städtische Landwirtschaft und Landnutzungsrecht am Beispiel Nairobis

Rita Schäfer

Hausgärten und Gartengruppen von Frauen in Zimbabwe und Sierra Leone

Nigel Swain

Traditionen der häuslichen Kleinlandwirtschaft in Osteuropa

Teodor Shanin

Dorfleben und informelle Ökonomie in Rußland

\section{ANDERS LEBEN}

Peter Gerber

Coopérative Européenne Longo Maï - Subsistenzwirtschaft und Selbstverwaltung 


\section{Edie Stone}

Community Gardening in New York City wird zur politischen Bewegung

Tetsuo Akemine, Richard Pestemer

Die Selbstversorgungs-Guerilla in Japan

Wolfgang Eisenberg

Zur politischen Bedeutung der Selbstversorger-Landwirtschaft im Wendland

\section{LEBENSKEIME}

\section{Farida Akhter}

Nayakrishi Andolon - Eine Kleinbauern Bewegung in Bangladesch

Maria Mies

Weltweite Proteste gegen die Globalisierung: „Seattle“

und „La Via Campesina“

Andrea Heistinger

Saatkunst und Kulturpflanzen von Bäuerinnen in Südtirol.

Christian Hiß

Kleinlandwirtschaft als Notwendigkeit für die Erhaltung der Biodiversität

Thomas Gladis

Immigrantengärten in Bonn - Vielfalt der Kulturen

\section{DAS GUTE LEBEN}

\section{Sigrid Fronius}

Ein Gartenprojekt in den subtropischen Anden

Brigitte N. Vogl-Lukasser, Christian R. Vogl, Harald Bohlar-Nordenkampf

Bäuerliche Hausgärten in Osttirol

Julia Kemna

Gartenbautherapie - von der heilenden Kraft der Gärten

Gert Gröning

Gemeinschaftsgärten in Nordamerika

Elisabeth Meyer-Renschhausen

Gärten und landwirtschaftliche Projekte in Berlin und Brandenburg ein Exkursionsbericht 


\section{Vorwort}

Die Arbeitsgruppe „Kleinstlandwirtschaft und Gärten in Stadt und Land“ entstand, als Johanna Machens, Torsten Reinsch, Renate Müller und Elisabeth Meyer-Renschhausen bei gemeinsamer Feldforschung in der Uckermark 1997 die soziale Bedeutung der Kleinstlandwirtschaft vor allem für Frauen entdeckten. Daraus entwickelte sich ab Wintersemester 1998/99 ein Forschungskolloquium. Von vielen Interessierten wurden feste Mitglieder der Arbeitsgruppe Petra Becker, Christophe Kotanyi, Frithjof Reul, Irmtraud Grünsteidel, Anne Holl, Julia Kemna sowie seitens der Hochschullehrer von der Landwirtschaftlich-Gärtnerischen Fakultät der Humboldt-Universität zu Berlin Friedhelm Streiffeler und Hardine Knuth sowie Gert Gröning von der Hochschule der Künste, Berlin. Das inhaltlich arbeitende Kolloquium entwickelte sich rasch auch zu einer Konferenzvorbereitungs-Gruppe. Die internationale Tagung Perspektiven der Kleinstlandwirtschaft und Gärten in Stadt und Land - zur sozialen und ökologischen Notwendigkeit einer ,weiblichen Ökonomie" konnte als eine Art Sommer-Fest mit weit über 100 Teilnehmenden vom 21.-25. Juli 2000 tatsächlich stattfinden in den Räumen der Landwirtschaftlich-Gärtnerischen Fakultät der HumboldtUniversität. Die Tagung wurde gefördert vom Förderprogramm Frauenforschung der Senatsverwaltung für Arbeit, Soziales und Frauen, Berlin, der SchweisfurthStiftung, München, der Deutschen Gesellschaft für internationale Entwicklung, Feldafing, und dem Arbeitskreis wissenschaftlich und künstlerisch tätiger Frauen e.V., Berlin, sowie dem Landesverband Gartenbau und Landwirtschaft BerlinBrandenburg e.V.

$\mathrm{Da}$ wir - über weite Strecken ehrenamtlich und „ohne Ausstattung“ arbeitend - durchhalten konnten, verdanken wir besonders der Frauenforschung an der - und allgemein der - Landwirtschaftlich-Gärtnerischen Fakultät, die sich für die Einrichtung der entsprechenden Professur für Frauenforschung in den Agrarwissenschaften eingesetzt hatte, in deren Rahmen die Feldforschungsseminare unternommen worden waren, besonders Parto Teherani-Krönner und Uta AltmannHoffmann. Wolfgang Bokelmann und andere halfen uns durch das Überlassen eines Büros in der leerstehenden Villa der Fakultät. Schließlich half uns auch die Abteilung Kultursoziologie des Instituts für Soziologie der Freien Universität Ber- 
lin, die uns - dank der Begeisterung von Dietmar Kamper für unser Anliegen -im letzten halben Jahr ein Büro überließ.

Mit diesem Buch legen wir einen Beitrag zur sozialen Bedeutung des neuen Phänomens städtischer und ländlicher Gärten und Kleinlandwirtschaften weltweit vor. Es handelt sich nicht einfach um eine pure Tagungsdokumentation. Einige der Beiträge wurden erst nach der Tagung eigens für dieses Buch geschrieben. Diejenigen Vorträge auf der Konferenz, die bereits im ersten Band zum Thema, „Die Wiederkehr der Gärten - Kleinlandwirtschaft im Zeitalter der Globalisierung", hrsg. von Elisabeth Meyer-Renschhausen und Anne Holl, Innsbruck: Studienverlag 2000, publiziert wurden, sind hier nicht wieder aufgenommen worden.

Das Besondere der Tagung war, daß hier Praktiker und Wissenschaftler gemeinsam diskutierten. Da viele unserer Autoren eher praktisch denn theoretisch tätig sind, bekamen wir manche Beiträge direkt aus dem Garten als Rohprodukt geschickt, die Erde klebte sozusagen noch dran. Wir haben uns bemüht, die Aufsätze nach bestem „Wissen und Gewissen“ zu einer für jedefrau und jedermann genießbaren Speise zuzubereiten.

Berlin, im Juli 2001

Elisabeth Meyer-Renschhausen, Renate Müller und Petra Becker 